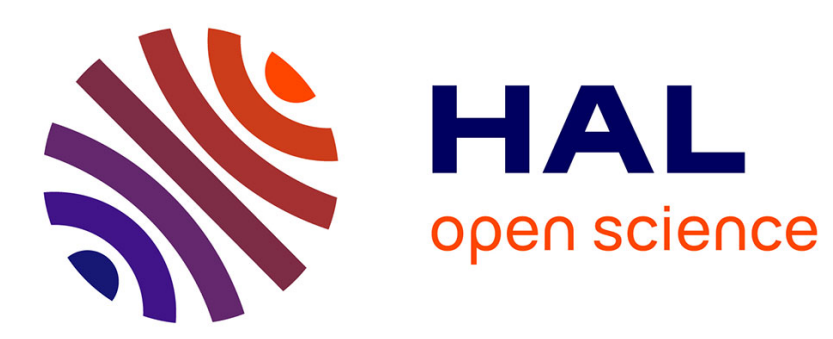

\title{
Irrationality is needed to compute with signal machines with only three speeds \\ Jérôme Durand-Lose
}

\section{To cite this version:}

Jérôme Durand-Lose. Irrationality is needed to compute with signal machines with only three speeds. 9th Conference on Computability in Europe 2013, Jul 2013, Milan, Italy. pp.108-119, 10.1007/978-3642-39053-1_12. hal-00807227

\section{HAL Id: hal-00807227 \\ https://hal.science/hal-00807227}

Submitted on 13 Jan 2017

HAL is a multi-disciplinary open access archive for the deposit and dissemination of scientific research documents, whether they are published or not. The documents may come from teaching and research institutions in France or abroad, or from public or private research centers.
L'archive ouverte pluridisciplinaire HAL, est destinée au dépôt et à la diffusion de documents scientifiques de niveau recherche, publiés ou non, émanant des établissements d'enseignement et de recherche français ou étrangers, des laboratoires publics ou privés. 


\title{
Irrationality is needed to compute with signal machines with only three speeds
}

\author{
Jérôme Durand-Lose* \\ LIFO, Université d'Orléans, \\ B.P. 6759, F-45067 ORLÉANS Cedex 2, France.
}

\begin{abstract}
Space-time diagrams of signal machines on finite configurations are composed of interconnected line segments in the Euclidean plane. As the system runs, a network emerges. If segments extend only in one or two directions, the dynamics is finite and simplistic. With four directions, it is known that fractal generation, accumulation and any Turing computation are possible.

This communication deals with the three directions/speeds case. If there is no irrational ratio (between initial distances between signals or between speeds) then the network follows a mesh preventing accumulation and forcing a cyclic behavior. With an irrational ratio (here, the Golden ratio) between initial distances, it becomes possible to provoke an accumulation that generates infinitely many interacting signals in a bounded portion of the Euclidean plane. This behavior is then controlled and used in order to simulate a Turing machine and generate a 25 -state 3 -speed Turinguniversal signal machine.
\end{abstract}

Key-words. Accumulation; Computability; Fractal; Model of computation; Signal machine; Turing machine; Unconventional computation.

\section{Introduction}

Imagine yourself with some color pencils and a sheet of paper together with ruler and compass. Some colored line segments are drawn and you are given rules so as to extend the drawing. According to the rules and the initial drawing/configuration you might stop soon, have to extend the paper indefinitely or draw forever in a bounded part of the paper as on top of Fig. 1(a). Could such a system compute?

This communication concentrates on the case where the dynamical system is a signal machine. In this setting, one drawing direction is distinguished and used as time axis. Line segments are enlarged synchronously until they intersect one another and get replaced. This goes on until no more collision can happen.

The line segments are the traces of signals and their intersections are collisions. Each signal corresponds to some meta-signal. During a collision, in-coming

* This work was partially funded by the ANR project AGAPE, ANR-09-BLAN-015903. Jerome.Durand-Lose@univ-orleans.fr 


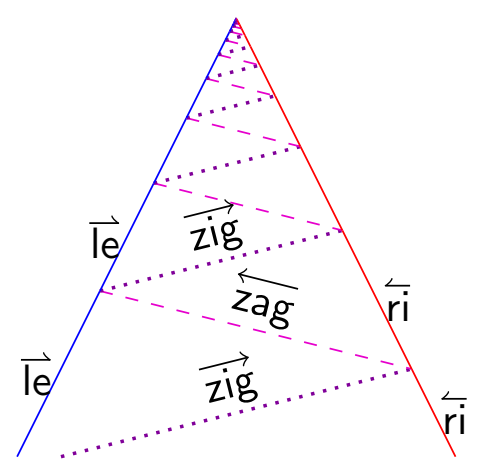

(a) Most basic accumulation.

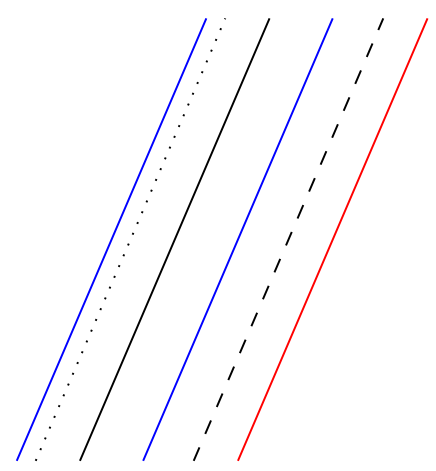

(b) One speed: no collision.

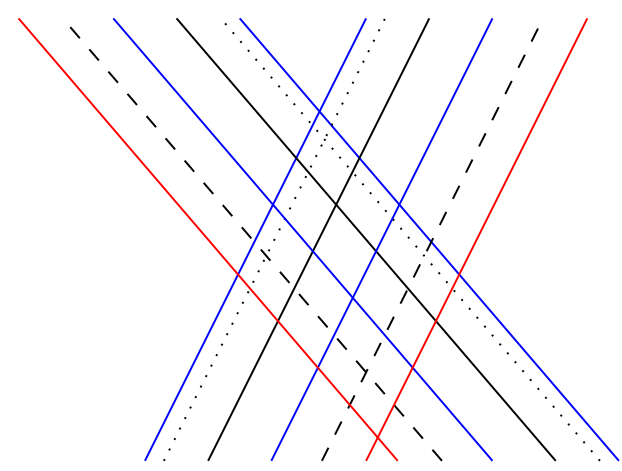

(c) Two speeds:

finitely many collisions.

Fig. 1. Basic cases.

signals are removed and new ones are emitted according to the meta-signals associated to the incoming signals. This is called a collision rule. Signals that correspond to the same meta-signal must travel at the same speed (or directions on the drawing) thus the resulting traces are parallel. There are finitely many meta-signals so there are finitely many collision rules.

The signals move on a one dimensional Euclidean space orthogonal to the temporal axis. Considering the traces leads to two dimensional drawings called space-time diagrams (as illustrated throughout the communication). Space and time are continuous $\left(\mathbb{R} \times \mathbb{R}^{+}\right)$. Signals as well as collisions are dimensionless points. Computations are exact, there is no noise nor approximation.

Problematics. Signal machines are very powerful and colorful complex systems capable of computing in the classical Turing understanding [1]. This communication is in the line of minimality thresholds in order to have Turing-computability capability (like $[2,3]$ for Turing machines and $[4,5]$ for cellular automata; a more general picture is presented in [6]). This communication extends [7] on small Turing-universal signal machines (few meta-signals but $4+$ speeds) and [8] that addresses only accumulation (i.e. not computation) with 3 -speed signal machines.

Accumulations are easy to produce and are the cornerstone to hyper-computation in the model [9]. The present communication investigates the minimal number of speeds so that accumulations or Turing computations are possible (starting from a finite configuration). Four meta-signals of different speeds are enough to make an accumulation as depicted in Fig. 1(a). Four speeds and 15 meta-signals are enough to compute [7].

In this communication, only the number of different speeds is considered and in particular the case of three speeds. One speed does not allow any collision (see Fig. 1(b)). With two speeds, the number of collisions is finite and signals have to follow a regular grid which has no accumulation (see Fig. 1(c)). Three-speed signal machines with rational speeds and rational initial positions always enter a cyclic behavior with no accumulation and limited computing capability. But if an irrational ratio between distances in the initial configuration is allowed, then accumulations are possible as well as any Turing computation. 
State of the art. Signal machines are one of the unconventional models of computation dealing with Euclidean geometry together with Euclidean abstract machines [10,11], Piecewise Constant Derivatives systems [12] and colored universes [13].

Signal machines were originally introduced as a continuous counterpart of (discrete) cellular automata to provide a context for the underlying Euclidean (continuous) reasoning often found in the literature as well as to propose an abstract formalization of the concept of signal [14-16].

Accumulations provide a powerful tool to accelerate a computation, to do hyper-computation and analog computation [9]. Fractals can be generated and their construction modified so as to achieve massive parallelism and the capability to solve efficiently NSPACE-complete problems (Q-SAT in [17]).

In [8], the present author and colleagues already proved that irrational ratio between speeds or between initial distances is needed in order to have an accumulation with three speeds. They exhibit a geometrical implantation of the Euclid algorithm inside the computation. If all ratios are rational then this algorithm stops (generating a non-accumulating mesh) otherwise it goes on indefinitely provoking the accumulation. They also cover the case of an irrational ratio between speeds which is not addressed here.

Contribution. If all the ratios are rational, then some global regular mesh emerges. The signals have to be on that mesh which does not have any accumulation point. Moreover, being on this mesh ensures that the collisions have to be ultimately periodic so that the dynamics is ultimately cyclic and computing capability is limited. (The behavior is called cyclic and not periodic since at the configuration level, there is no periodicity.)

Using the Golden ratio between distances makes it possible to draw a fractal which accumulates. Because of self-similarity, ensuring one repetition step is enough.

With three speeds, it is straightforward to simulate a Turing machine on a bounded tape. But even though the tape remains finite, it cannot be bounded in the general case. From the fractal construction, a scheme is extracted to extend the tape on demand and get the full Turing computing power.

Outline. Section 2 provides all the definitions. Section 3 shows that in the rational case the dynamics is trapped into a mesh that does not allow any accumulation and restrain computing capability. Section 4 shows how to get an accumulation with non-rational ratio between distances. Section 5 provides the simulation of any Turing machine in such a case. Conclusion, remarks and perspectives are gathered in Sect. 6.

\section{Definitions}

This communication deals only with finite configurations so all definitions are restrained to this case. 
A signal machine collects the definitions of available meta-signals, their speeds (positive for rightward signals and negative for leftward ones) and their collision rules. For example, the machine to generate Fig. 1(a) is composed of the following meta-signals (with speed): $\overrightarrow{\mathrm{le}}\left(\frac{1}{2}\right), \overrightarrow{\mathrm{zig}}(4), \overleftarrow{\mathrm{zag}}(-4)$, and $\breve{\mathrm{ri}}\left(-\frac{1}{2}\right)$. There are only two collision rules:

$$
\{\overrightarrow{\mathrm{le}}, \overleftarrow{\mathrm{zag}}\} \rightarrow\{\overrightarrow{\mathrm{le}}, \overrightarrow{\mathrm{zig}}\} \quad \text { and } \quad\{\overrightarrow{\mathrm{zig}}, \overleftarrow{\mathrm{ri}}\} \rightarrow\{\overleftarrow{\mathrm{zag}}, \overleftarrow{\mathrm{ri}}\}
$$

It might happen that exactly three (or more) meta-signals meet. In such a case, collision rules involving three (or more) meta-signals are used. There can be any number of meta-signals in the range of a collision rule, as long as their speeds differ (i.e. they are not parallel).

Definition 1 (Signal machine) A Signal machine, $(M, S, R)$, is defined by: $M$ is a finite set of meta-signals, $S$ is a function from meta-signals to real numbers, assigning speeds, and $R$ is a deterministic set of collision rules. A collision rule is written $\rho=\rho^{-} \rightarrow \rho^{+}$where $\rho^{-}$and $\rho^{+}$are sets of meta-signals of different speeds, and $\rho^{-}$must have at least two meta-signals. The set of collision, rules, $R$ is deterministic: $\rho \neq \rho^{\prime}$ implies that $\rho^{-} \neq \rho^{\prime-}$.

A configuration is a function from the real line (space) into the set of metasignals and collision rules plus one extra value $\oslash$ (nothing there). There should be finitely many non- $\oslash$ locations.

Definition 2 (Configuration) A configuration, $c$, is a function from the real line into meta-signals, rules, and the value $\oslash$ (let $V=M \cup R \cup\{\oslash\}$ so that $c: \mathbb{R} \rightarrow V)$ such that $\left|c^{-1}(M \cup R)\right|<\infty$.

If there is a signal of speed $s$ at $x$, then, unless it enters a collision before, after a duration $\Delta t$, its position is $x+s \cdot \Delta t$. At a collision, all incoming signals are immediately replaced by outgoing signals in the following configurations according to collision rules.

Definition 3 (Sequence of collision times) Considering a configuration, $c$, the time to the next collision, $\Delta(c)$, is equal to the minimum of the positive real numbers $d$ such that:

$$
\exists x_{1}, x_{2} \in \mathbb{R}, x_{1} \neq x_{2}, \exists \mu_{1}, \mu_{2} \in M\left\{\begin{array}{l}
x_{1}+d \cdot S\left(\mu_{1}\right)=x_{2}+d \cdot S\left(\mu_{2}\right), \\
c\left(x_{1}\right)=\mu_{1} \vee\left(c\left(x_{1}\right)=\rho^{-} \rightarrow \rho^{+} \wedge \mu_{1} \in \rho^{+}\right), \\
c\left(x_{2}\right)=\mu_{2} \vee\left(c\left(x_{2}\right)=\rho^{-} \rightarrow \rho^{+} \wedge \mu_{2} \in \rho^{+}\right) .
\end{array}\right.
$$

It is $+\infty$ if there is no such $d$. The sequence of collision times is defined by: $t_{0}=0, t_{n+1}=t_{n}+\Delta\left(c_{t_{n}}\right)$ where $c_{t}$ denote the configuration at time $t$.

This sequence is finite if there is an $n$ such that $\Delta\left(c_{t_{n}}\right)=+\infty$. Otherwise, since it is non-decreasing, it admits a limit. If the sequence is finite or its limit is infinite, then the whole space-time diagram is defined. Otherwise, there is an accumulation and the limit configuration is left undefined.

Definition 4 (Dynamics between collisions) For $t^{\prime}$ between $t$ and $t+\Delta\left(c_{t}\right)$, the configuration at $t^{\prime}$ is defined as follows. Signals are set: $c_{t^{\prime}}\left(x^{\prime}\right)=\mu$ iff $c_{t}(x)=$ $\mu \vee\left(c_{t}(x)=\rho^{-} \rightarrow \rho^{+} \wedge \mu \in \rho^{+}\right.$) where $x=x^{\prime}+\left(t-t^{\prime}\right) \cdot S(\mu)$. (There is no collision to set.) It is $\oslash$ everywhere else. 
Definition 5 (Dynamics at a collision time) For the configuration at $t^{\prime}=$ $t+\Delta\left(c_{t}\right)$, collisions are set first: $c_{t^{\prime}}\left(x^{\prime}\right)=\rho^{-} \rightarrow \rho^{+}$iff for all $\mu \in \rho^{-}, c_{t}\left(x_{\mu}\right)=$ $\mu \vee\left(c_{t}\left(x_{\mu}\right)=\rho^{-} \rightarrow \rho^{+} \wedge \mu \in \rho^{+}\right)$where $x_{\mu}=x^{\prime}+\left(t-t^{\prime}\right) \cdot S(\mu)$. Then meta-signals are set (where there is not already a collision). It is $\oslash$ everywhere else.

A space-time diagram is the collection of consecutive configurations which forms a two dimensional picture. In the space-time diagram in Fig. 1(a) the sequence of collision times is given by the collisions of $\overrightarrow{\mathrm{zig}}$ on $\overleftarrow{\mathrm{ri}}$, then $\overleftarrow{\mathrm{zag}}$ on $\overrightarrow{\mathrm{le}}$, then $\overrightarrow{z i g}$ on $\overrightarrow{r i}$... This sequence accumulates on top of the space-time diagram.

Definition 6 (Rational signal machine) A signal machine is rational if all speeds are rational numbers and any non- $\oslash$ position in any initial configuration must also be a rational number.

Since the position of collisions are solutions of systems of rational linear equations, they are rational. (Coordinates of accumulation may be non-rational [18].)

Definition 7 (Rational-like) A signal machine is rational-like if its speeds are rational up to a coefficient. (There is no restriction on possible initial configuration.) A configuration is rational-like if all the ratios between distances between signals are rational.

Linear transformation. It is possible to linearly change the speed of the metasignals or the positions in the initial configuration. As long as the coefficient is positive, the dynamics is not changed. This comes from the absence of any absolute origin or scale. This is not formally proved (this was done, e.g., in [19, Chap. 5]), but exemplified by the space-time diagrams in Fig. 2.

A linear transformation with a positive ratio of all the positions in the initial configuration only corresponds to changing the spatial origin and the space scale (Fig. 2(a)), time scale has to be changed accordingly.

A linear transformation with a positive ratio of all the speeds of the signal machine results in a change in the scale of time (but not of space, Fig. 2(b)). The term added corresponds to a drift, i.e. a slant in the time axis (Fig. 2(c)).

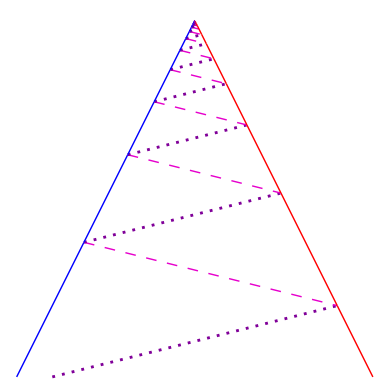

(a) Initial positions divided by 2 .

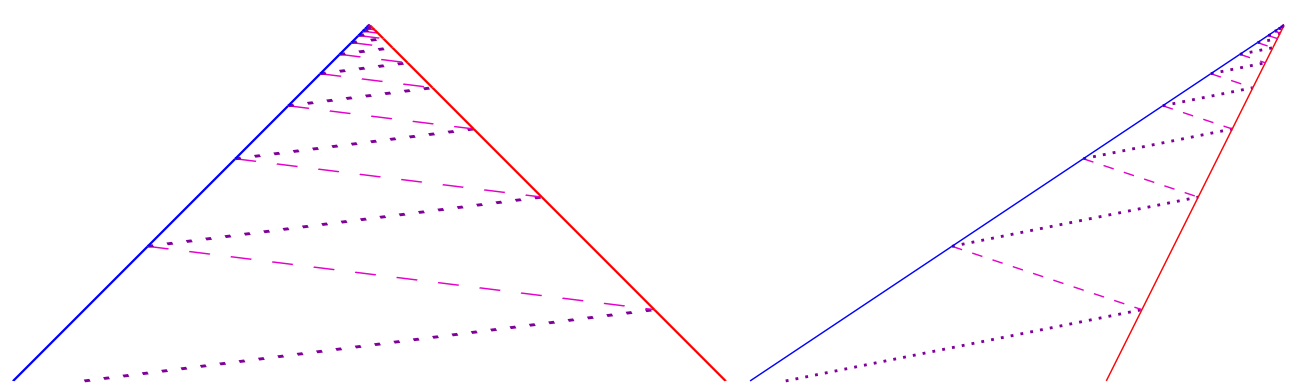

(b) Speeds multiplied by 2 .

(c) Speeds multiplied by 2 plus 1.

Fig. 2. Linear transformations on the space-time diagram of Fig. 1(a). 
Only locations are affected. The existence of a collision as well as the computing capability does not depend on linear transformations with positive ratio.

Normalization. When considering a finite set of real numbers, there is always a unique linear transformation with positive ratio that maps the two lowest values in the set to 0 and 1 . We call this a 0-1-normalization.

If all ratios between distances in a configuration are rational then its 0-1normalization has only signals at rational coordinates: with $O$ and $I$ the signals with new coordinate 0 and 1 , a signal at position $M$ have coordinate $\overline{O M} / \overline{O I}$ which is rational since non-degenerated linear operators preserve ratio.

That ratios between distances are rational is both a sufficient and necessary condition to rescale into $\mathbb{Q}$. Up to normalization, the dynamics of a rational-like signal machine on a rational-like configuration is the one of a rational signal machine.

\section{Rational 3-speed signal machines}

If speeds are rational up to a coefficient, then their 0-1-normalization results in rational speeds. The 0 -1-normalized speeds are 0,1 , and (a rational value greater than 1) $1+p / q$ (with $p, q \in \mathbb{N}, 1 \leq p, q$ and relatively prime). They are linearly transformed into $-q, 0$ and $p$.

The 0-1-normalized initial configuration is scaled so that all (signal) positions are natural numbers. The extreme positions are 0 and $n$.

Definition $8((p, q, n)$-mesh) The $(p, q, n)$-mesh corresponds to the union of the following half-lines of $\mathbb{R} \times \mathbb{R}^{+}$:

- $V_{v}: x=v /(p+q)$ where $v \in\{0,1,2, \ldots, n(p+q)\}$,

- $L_{l}: x \leq n$, and $x+q . t=l / p$ where $l \in\{0,1,2, \ldots\}$ and

$-R_{r}: 0 \leq x$ and $x-p . t=r / q$ where $r \in\{\ldots,-1,0,1,2, \ldots,(n . q)\}$.

Figure 3 shows the display of such a mesh. Half-lines $V_{v}$ are vertical, $L_{l}$ have a negative slope and are dotted and $R_{r}$ have a positive slope and are dashed.

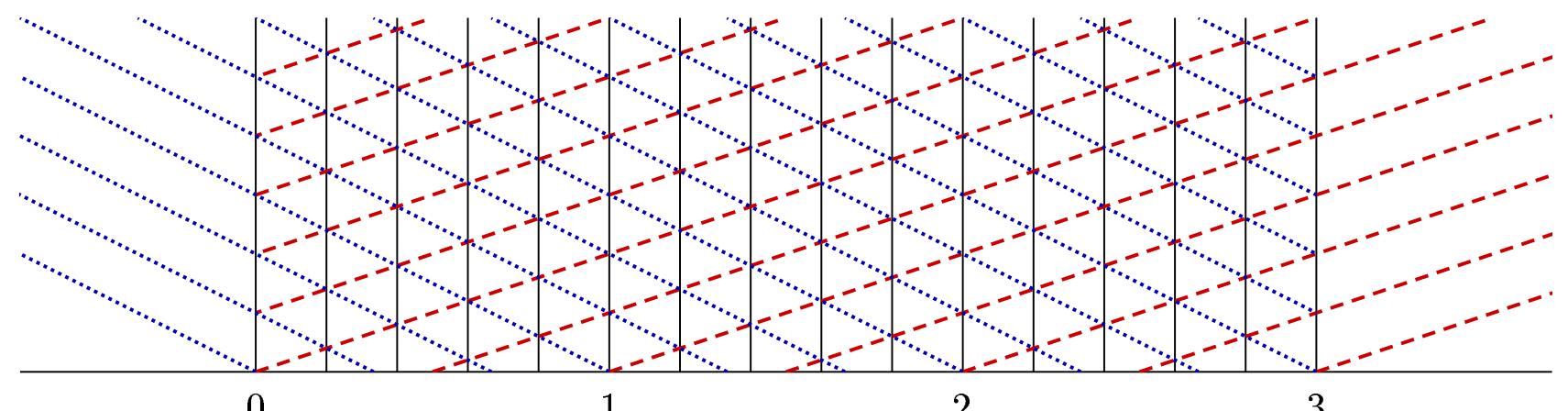

0

2

3

Fig. 3. (3, 2, 3)-mesh.

A mesh has only $L_{l}$ half-lines when $x<0$ and only $R_{r}$ half-lines when $n<x$. When $0 \leq x \leq n$, then all three kinds of lines are present and each every point 
of intersection is incident upon a line of each type. Moreover, there is a line of each kind starting at positions $(x, 0)$ when $x \in\{0,1,2, \ldots, n\}$.

Lemma 1. During the computation of a rational machine with speeds $-q, 0$ and $p$ from an initial configuration with no signal outside of $\{0,1,2, \ldots, n\}$, there is no signal outside the mesh $(p, q, n)$.

This is true because: initially signals are on the mesh on a half-line with the same speed/slope (all are issued from coordinates in $\{0,1,2, \ldots, n\}$ ); if a signal is on such half-lines, it remains upon it until it participates in a collision; and new signals appear only in collisions, collisions can only happen at line crossings and all three kinds of lines go on from there.

If the computation would lead to an accumulation, then this accumulation should also be on the mesh. But the mesh has no accumulation.

The mesh is time periodic in the region $0 \leq x \leq n$ and the computation in this part does not receive anything from the outside. When considering the configurations at time where lines intersect on $x=0$, the mesh is exactly the same. On $0 \leq x \leq n$, these configurations can be described by a fixed length string on meta-signals and collision rules. Since this alphabet is finite (and the system is deterministic), this eventually enters a cycle.

Outside of the region $0 \leq x \leq n$, the mesh is also ultimately time-periodic. No collision happens there and the output signals also eventually enter a cycle. Rational 3-speed signal machines always enter a cyclic behavior. Transient time and cycle duration can be computed from the initial configuration and machine. These bounds prevent Turing-universality.

Lemma 2. Rational 3-speed signal machines cannot produce accumulation and are not Turing-universal.

This also holds for rational-like machines on rational-like initial configurations.

\section{Accumulation with the Golden ratio}

Using the Golden ratio, it is possible to generate the accumulating fractal in Fig. 4(a). The signal machine is directly read from the picture as depicted in Fig. 4(b). The signal machine is rational-like but not rational since the initial configuration is not.

The initial configuration is not part of the fractal cycle since we prefer to have all signals but one parallel. The distance between cell and seed is taken to be one. The exact position of $\overrightarrow{\text { right }}$ between cell and seed is not important. To position border, $\varphi$ has to be given a value so that the fractal is generated, i.e. the ratio of the distance of the three last signals is preserved.

As indicated in Fig. 4(b), the first ratio is $\frac{\varphi}{1}$. To compute the next ratio, the distance between the third cell and border has to be 1 because of the presence of the parallelogram (with left and $\overrightarrow{\text { right }}$ sides) and the parallelism of the cell and 


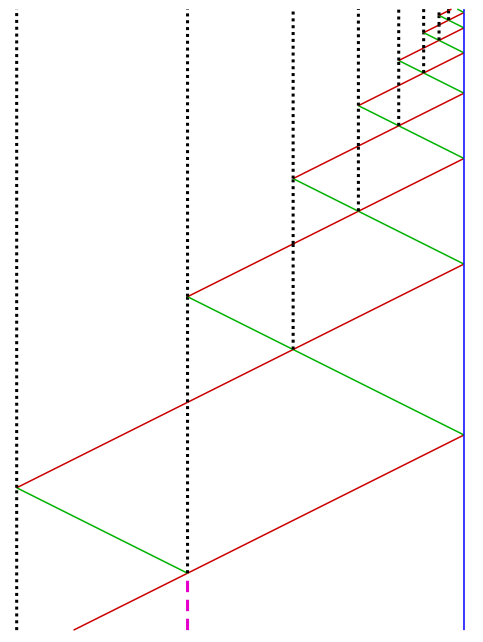

(a) Fractal

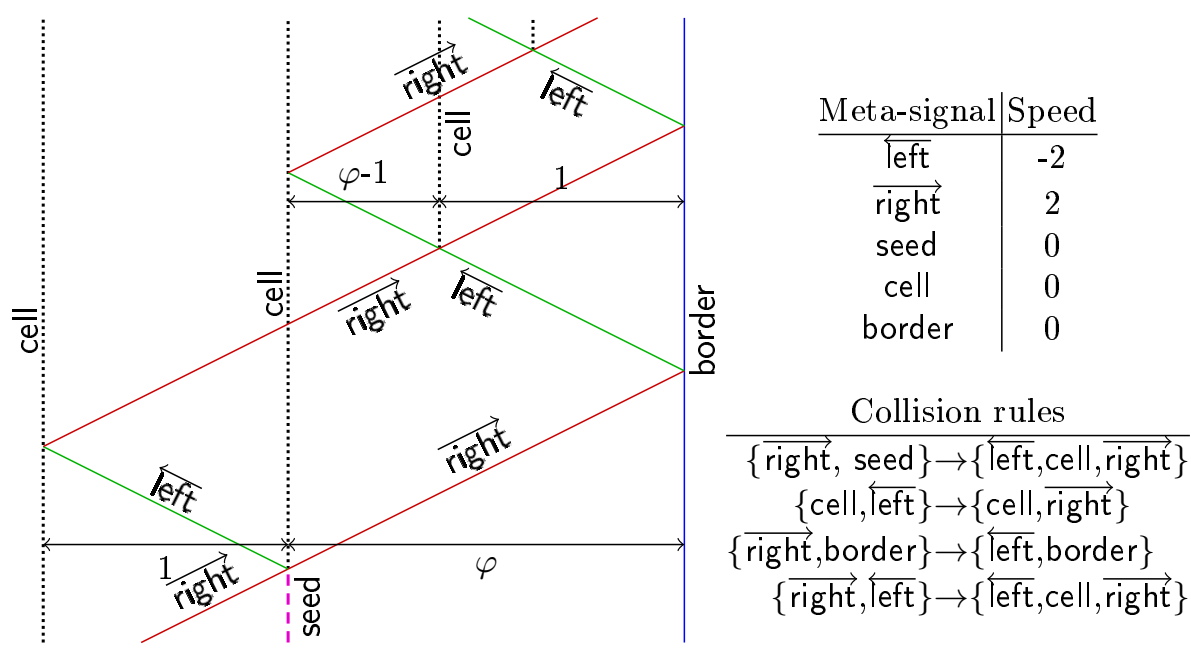

(b) Fractal construction

Fig. 4. Accumulating with three speeds and an irrational position.

border signals. Thus the other distance has to be $\varphi-1$. The second ratio is $\frac{1}{\varphi-1}$. By equaling these ratios, $\varphi$ is $\frac{1+\sqrt{5}}{2}$, the Golden ratio.

The construction then repeats forever. The ratio is preserved and the fractal is generated.

Lemma 3. A 3-speed signal machine started on an irrational configuration can generate an accumulation, even when all speeds are rational.

In fact, this is for any non-rational ratio. Moreover, any non-rational ratio between speeds can also be "transferred" into distances so that an accumulation can be produced [8].

\section{Computing with the Golden ratio}

It is possible to start simulating a Turing machine with a rational 3-speed signal machine. Null speed signals are used to encode the cells of the tape. Each one encodes a symbol of the tape (\# is the blank symbol). The head is encoded by a sequence of signals that record the state and move left or right (with the two other speeds). Each time it collides on a "symbol" signal, it updates the cell and the state and then goes left or right. This is done according to the transition table of the Turing machine. The meta-signals and collision rules are given on Fig. 5. An example of this simulation is given on the lower half of Fig. 6 .

The tape is finite but not bounded. Starting from a finite configuration, the representation of the tape should be enlargeable. The fractal presented in previous section allows one to generate an unbounded sequence of sites (purposely named cell) to simulate the tape of a Turing machine. It is not fully generated to avoid an accumulation. A new cell is generated only when the tape needs enlargement.

An enlargement can be seen on the upper half of Fig. 6(b). It is started when a rightward state-encoding signal $\left(\overrightarrow{q_{0}}\right.$ in the figure) reaches the border signal, 


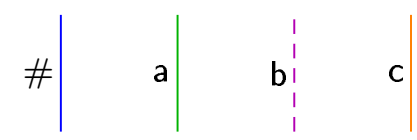

\begin{tabular}{c|c} 
Meta-signal & Speed \\
\hline$\#, a, b, c$ & 0
\end{tabular}

(a) Symbols $\{\#, a, b, c\}$

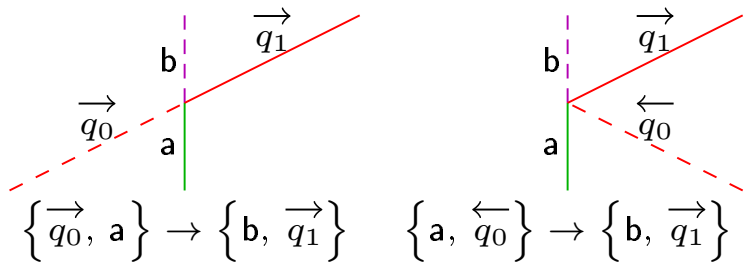

(b) Transition case $\delta\left(q_{0}, \mathrm{a}\right)=\left(q_{1}, \mathrm{~b}, \rightarrow\right)$
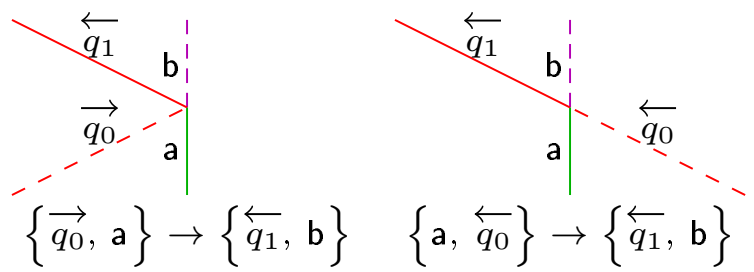

(d) Transition case $\delta\left(q_{0}, \mathrm{a}\right)=\left(q_{1}, \mathrm{~b}, \leftarrow\right)$

Fig. 5. Basic encoding of a Turing machine.

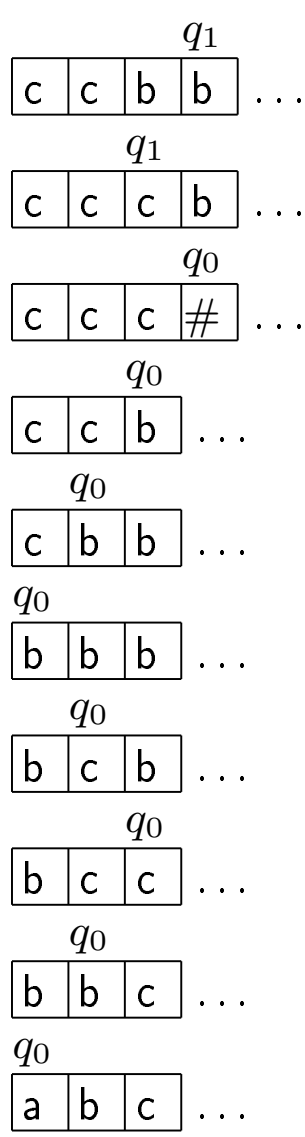

(a) Turing machine run

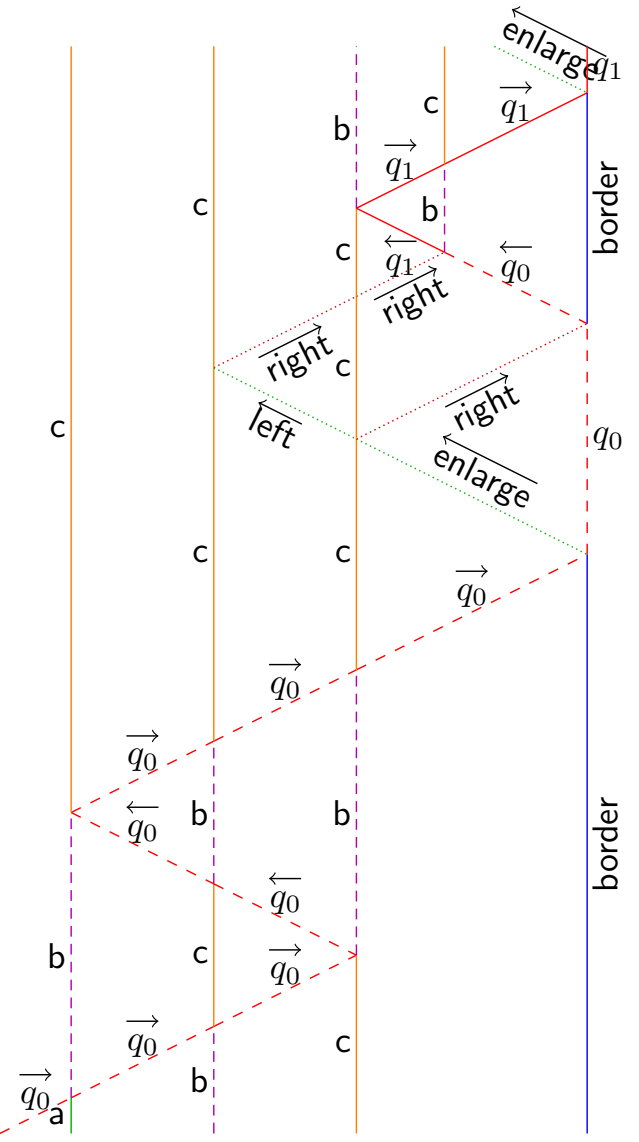

(b) Labeled simulation

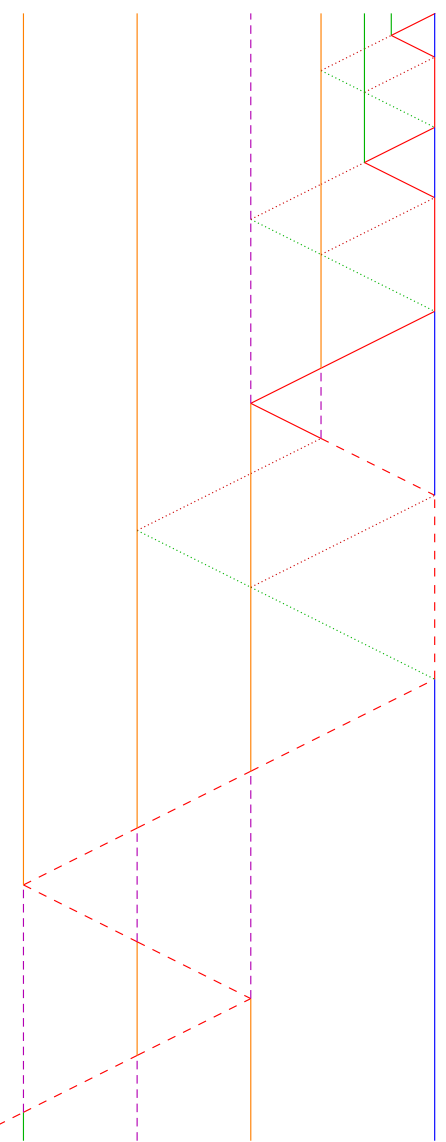

(c) Longer simulation

Fig. 6. Computing with three speeds and an irrational position.

this means that the head is looking for a cell that does not exist yet. Signal border is replaced by a motionless signal encoding the state ( $q_{0}$ in the figure)

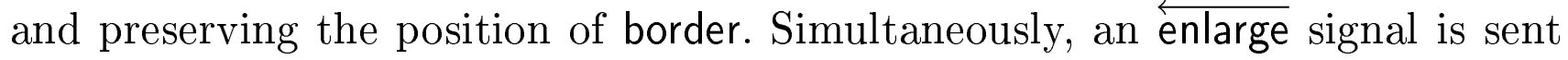
on the left to create the new cell. 
This creation uses the same parallelogram construction as before (dotted

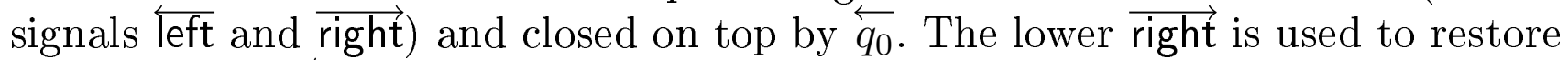
border and set $\overleftarrow{q_{0}}$ on movement. The latter, instead of colliding with a symbol signal, collides with the upper $\overrightarrow{\text { right. }}$. The collision happens as if it were on the blank symbol. The needed meta-signals and collision rules are given in Fig. 7 .

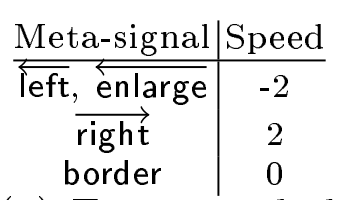

(a) Extra symbols

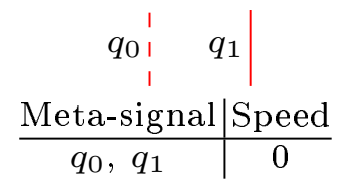

(d) States $\left\{q_{0}, q_{1}\right\}$

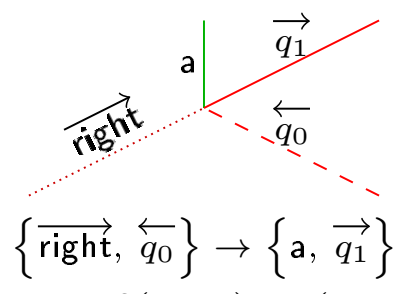

(b) Case $\delta\left(q_{0}, \#\right)=\left(q_{1}, \mathrm{a}, \rightarrow\right)$

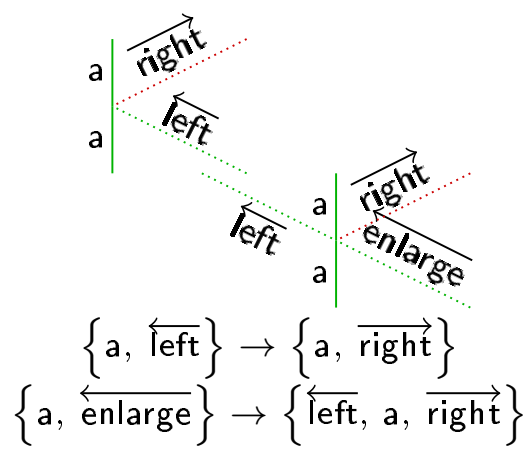

(e) For each symbol a

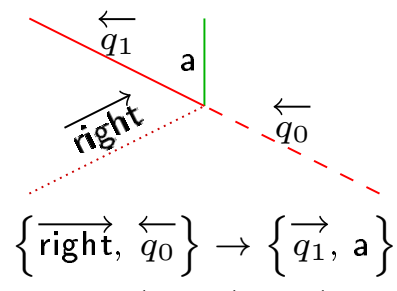

(c) Case $\delta\left(q_{0}, \#\right)=\left(q_{1}, \mathrm{a}, \leftarrow\right)$

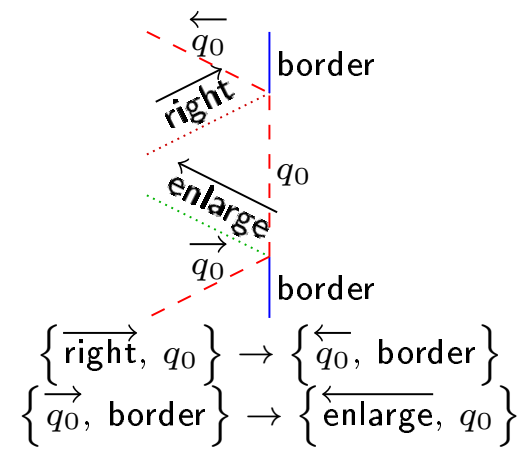

(f) For each state $q_{0}$

Fig. 7. Extra encoding to enlarge the simulation.

\section{Conclusion and perspectives}

With three speeds, it is possible to accumulate only if there is an irrational ratio between initial positions or between speeds (see [8] for speeds).

The number of meta-signals for the Turing-machine simulation with the Golden ratio is 1 for each symbol plus 3 for each state plus 4 . With the small universal Turing machines listed in [3], a Turing-universal 25-meta-signal 3-speed signal machine using a Golden ratio distance can be constructed.

With a Golden ratio location, undecidable problems arise. Directly from classical computability theory, to have only finitely many collisions (or to enter a cyclic behavior) is not decidable. In the simulation of a Turing machine, if the head goes on the right each time, then an accumulation is generated. For an accumulation to happen, the head would have to explore all the cells on the right which is also not decidable. (In the general case, forecasting an accumulation is $\Sigma_{2}^{0}$-complete [20].)

An irrational ratio is an important piece of information (it could encode the halting problem). We conjecture that it is possible to use it as an oracle. 
In [9], accumulations are used in order to hyper-compute, with an irrational ratio, computations and accumulations become possible, we conjecture that it is possible to solve the halting problem and even to climb the finite levels of the arithmetic hierarchy with three speeds and the Golden ratio.

Signal machines are know to be able to do analog computations [9] with real numbers encoded in distances. Up to what extent is analog computation possible with three speeds?

\section{References}

1. Durand-Lose, J.: Abstract geometrical computation: Turing computing ability and undecidability. In Cooper, B.S., Löwe, B., Torenvliet, L., eds.: New Computational Paradigms, 1st Conf. Computability in Europe (CiE '05). Number 3526 in LNCS, Springer (2005) 106-116

2. Rogozhin, Y.V.: Small universal Turing machines. Theoret. Comp. Sci. 168 (1996) 215-240

3. Woods, D., Neary, T.: The complexity of small universal Turing machines: A survey. Theoret. Comp. Sci. 410 (2009) 443-450

4. Cook, M.: Universality in elementary cellular automata. Complex Systems $\mathbf{1 5}$ (2004) $1-40$

5. Ollinger, N., Richard, G.: Four states are enough! Theoret. Comp. Sci. 412 (2011) $22-32$

6. Margenstern, M.: Frontier between decidability and undecidability: a survey. Theor. Comput. Sci. 231 (2000) 217-251

7. Durand-Lose, J.: Abstract geometrical computation 4: small Turing universal signal machines. Theoret. Comp. Sci. 412 (2011) 57-67

8. Becker, F., Chapelle, M., Durand-Lose, J., Levorato, V., Senot, M.: Abstract geometrical computation 8: Small machines, accumulations \& rationality. Draft (2013)

9. Durand-Lose, J.: Abstract geometrical computation 3: Black holes for classical and analog computing. Nat. Comput. 8 (2009) 455-472

10. Huckenbeck, U.: Euclidian geometry in terms of automata theory. Theoret. Comp. Sci. 68 (1989) 71-87

11. Mycka, J., Coelho, F., Costa, J.F.: The euclid abstract machine: Trisection of the angle and the halting problem. In Calude, C.S., Dinneen, M.J., Păun, G., Rozenberg, G., Stepney, S., eds.: Unconventional Computation, 5th Int. Conf., UC '06, York, UK, September 4-8, 2006, Proceedings. Number 4135 in LNCS, Springer (2006) 195-206

12. Bournez, O.: Some bounds on the computational power of piecewise constant derivative systems (extended abstract). In: ICALP '97. Number 1256 in LNCS (1997) 143-153

13. Jacopini, G., Sontacchi, G.: Reversible parallel computation: an evolving spacemodel. Theoret. Comp. Sci. 73 (1990) 1-46

14. Mazoyer, J.: Computations on one dimensional cellular automata. Ann. Math. Artif. Intell. 16 (1996) 285-309

15. Mazoyer, J., Terrier, V.: Signals in one-dimensional cellular automata. Theoret. Comp. Sci. 217 (1999) 53-80 
16. Durand-Lose, J.: The signal point of view: from cellular automata to signal machines. In Durand, B., ed.: Journées Automates cellulaires (JAC '08). (2008) 238249

17. Duchier, D., Durand-Lose, J., Senot, M.: Computing in the fractal cloud: modular generic solvers for SAT and Q-SAT variants. In Agrawal, M., Cooper, B.S., Li, A., eds.: Theory and Applications of Models of Computations (TAMC '12). Number 7287 in LNCS, Springer (2012) 435-447

18. Durand-Lose, J.: Abstract geometrical computation 7: Geometrical accumulations and computably enumerable real numbers. Nat. Comput. 11 (2012) 609-622 Special issue on Unconv. Comp. ' 11.

19. Durand-Lose, J.: Calculer géométriquement sur le plan - machines à signaux. Habilitation à Diriger des Recherches, École Doctorale STIC, Université de NiceSophia Antipolis (2003) In French.

20. Durand-Lose, J.: Forcasting black holes in abstract geometrical computation is highly unpredictable. In Cai, J.Y., Cooper, B.S., Li, A., eds.: Theory and Applications of Models of Computations (TAMC '06). Number 3959 in LNCS, Springer (2006) 644-653 